\title{
Oil-source rock correlation and quantitative assessment of Ordovician mixed oils in the Tazhong Uplift, Tarim Basin
}

\author{
Li Sumei ${ }^{1 *}$, Pang Xiongqi ${ }^{1}$, Zhang Baoshou ${ }^{2}$, Xiao Zhongyao ${ }^{2}$ and Gu \\ Qiaoyuan $^{2}$
}

${ }^{1}$ State Key Laboratory of Petroleum Resources and Prospecting, China University of Petroleum, Beijing 102249, China

${ }^{2}$ Tarim Oilfield Company, PetroChina, Korla, Xinjiang 841000, China

(c) China University of Petroleum (Beijing) and Springer-Verlag Berlin Heidelberg 2010

\begin{abstract}
The origin of the marine oils in the Tarim Basin has long been a disputed topic. A total of 58 DST (drill stem test) crude oil and 8 rock samples were investigated using a comprehensive geochemical method to characterize and identify the origin of the Ordovician oils in the Tazhong Uplift, Tarim Basin, northwest China. Detailed oil-oil and oil-source rock correlations show that the majority of the oils have typical biomarker characteristics of the Middle-Upper Ordovician $\left(\mathrm{O}_{2+3}\right)$ source rock and the related crude oil. These characteristics include a distinct "V-shaped" relative abundance of $\mathrm{C}_{27}, \mathrm{C}_{28}$ and $\mathrm{C}_{29}$ regular steranes, low abundance of dinosterane, 24-norcholestanes, triaromatic dinosteroids and gammacerane. Only a few oils display typical biomarker characteristics indicating the Cambrian-Lower Ordovician $\left(\in-\mathrm{O}_{1}\right)$ genetic affinity, such as linear or anti " $\mathrm{"} \mathrm{shape} \mathrm{distribution} \mathrm{of} \mathrm{C}_{27}, \mathrm{C}_{28}$ and $\mathrm{C}_{29}$ regular sterane, with relatively high concentrations of dinosterane, 24-norcholestanes, triaromatic dinosteroids and gammacerane. It appears that most of the Ordovician oils in the Tazhong Uplift were derived from the $\mathrm{O}_{2+3}$ intervals, as suggested by previous studies. However, the compound specific $n$-alkane stable carbon isotope data indicate that the Ordovician oils are mixtures from both the $\in-\mathrm{O}_{1}$ and $\mathrm{O}_{2+3}$ source rocks rather than from the $\mathrm{O}_{2+3}$ strata alone. It was calculated that the proportion of the $\in-\mathrm{O}_{1}$ genetic affinity oils mixed is about $10.8 \%-74.1 \%$, with an increasing trend with increasing burial depth. This new oil-mixing model is critical for understanding hydrocarbon generation and accumulation mechanisms in the region, and may have important implications for further hydrocarbon exploration in the Tarim Basin.
\end{abstract}

Key words: Biomarker, compound specific isotope, oil-source rock correlation, mixed oil

\section{Introduction}

Ordovician carbonate is the most important hydrocarbonproducing formation in the Tazhong Uplift, Tarim Basin, and most of oil is discovered in the Middle-Upper Ordovician in the No.1 Fault Belt. Recently, commercial oils have been acquired from the Lower Ordovician, which suggests great hydrocarbon potential in deep strata of the area. Although large amounts of oil have been found in the Paleozoic reefflat complexes (Zhou et al, 2006), the source of the Paleozoic petroleum systems remains to be confirmed. This task has been hindered by the relatively high thermal maturity of the oils and the scarcity of deep wells drilled into the source rock intervals. Regardless, considerable progress has been made in identifying oil sources in the Tarim Basin in the last decade (Graham et al, 1990; Liang et al, 2000; Zhang et al,

*Corresponding author. email: smli8888@yahoo.com.cn Received September 2, 2009 2000a; 2000b; 2004). Zhang et al (2000a; 2000b) and Hanson et al (2000) deduced a Middle-Upper Ordovician source deposited in slope dysoxic or anoxic environments adjacent to a widespread carbonate platform margin based on ageindicating biomarkers. However, Sun et al (2003) argued that the Cambrian-Lower Ordovician strata might be important candidates for the source of the oils in the Tazhong and Tabei uplifts in the Tarim Basin according to aryl isoprenoids detected in the oils and source rocks in the basin. Cai et al (2007) and Chen et al (2008) suggested from sulfur isotope data that the Cambrian rocks might be the main source for the Tazhong oils. Additionally, some other researchers suggested mainly from biomarker evidence that mixed-source oils might exist in both the Tazhong and Tabei uplifts in the basin (Zhang et al, 2007; Guo et al, 2009; Zhao et al, 2008) and odd-even or even-odd carbon predominance of $n$-alkanes (Luo et al, 2007). Stable carbon isotope data of group composition was also used by Guo et al (2009) as a complementary approach in the oil-source identification in the area. 
In this study, we attempt to clarify the sources for the Ordovician oils in the Tazhong Uplift using compound specific isotope data of $n$-alkanes combined with biomarkers, and try to determine the proportion of sources contributing to the oils.

\section{Geological background}

The Tazhong Uplift in the Tarim Basin has a total area of approximately $30,000 \mathrm{~km}^{2}$. It is surrounded by the Manjiaer Depression in the north, the Tangguzibasi Depression in the south, the Awati Sag in the west, and the Tadong Sag in the east. The area boundary is on the Bachu Uplift in the southwest. The uplift is oriented NW-SE, and has a "broom" shape opening to the west and converging eastwards (Fig. 1(a)). It has five tectonic units, including the No.1 Fault Belt,
North Slope, Central Faulted Horst Belt and South Slope from north to south, and the East Burial Hill (Fig. 1). The general stratigraphic column of the Tazhong Uplift can be divided into several tectonostratigraphic sequences. The Cambrian section is composed of tidal platforms, platform marginal carbonates and evaporite rocks. The Middle Cambrian consists of a set of supratidal anhydrite-bearing dolomite developed in a large area of anhydrite and salt beds, which is about 200,000 $\mathrm{km}^{2}$ in area and 400-1,400 $\mathrm{m}$ in thickness (Cai et al, 2001). The Ordovician carbonate rocks are characterized by a deepening-upward and then shoaling-upward sequence within bioclastic grainstone, packstone and mudstone to sandstone. The Silurian sandstone sequence consists of tidal sandstone and brown or red mudstone. The Carboniferous sandstone consists of marine clastics, while the Mesozoic and Cenozoic are mainly composed of terrestrial sandstones and mudstones.

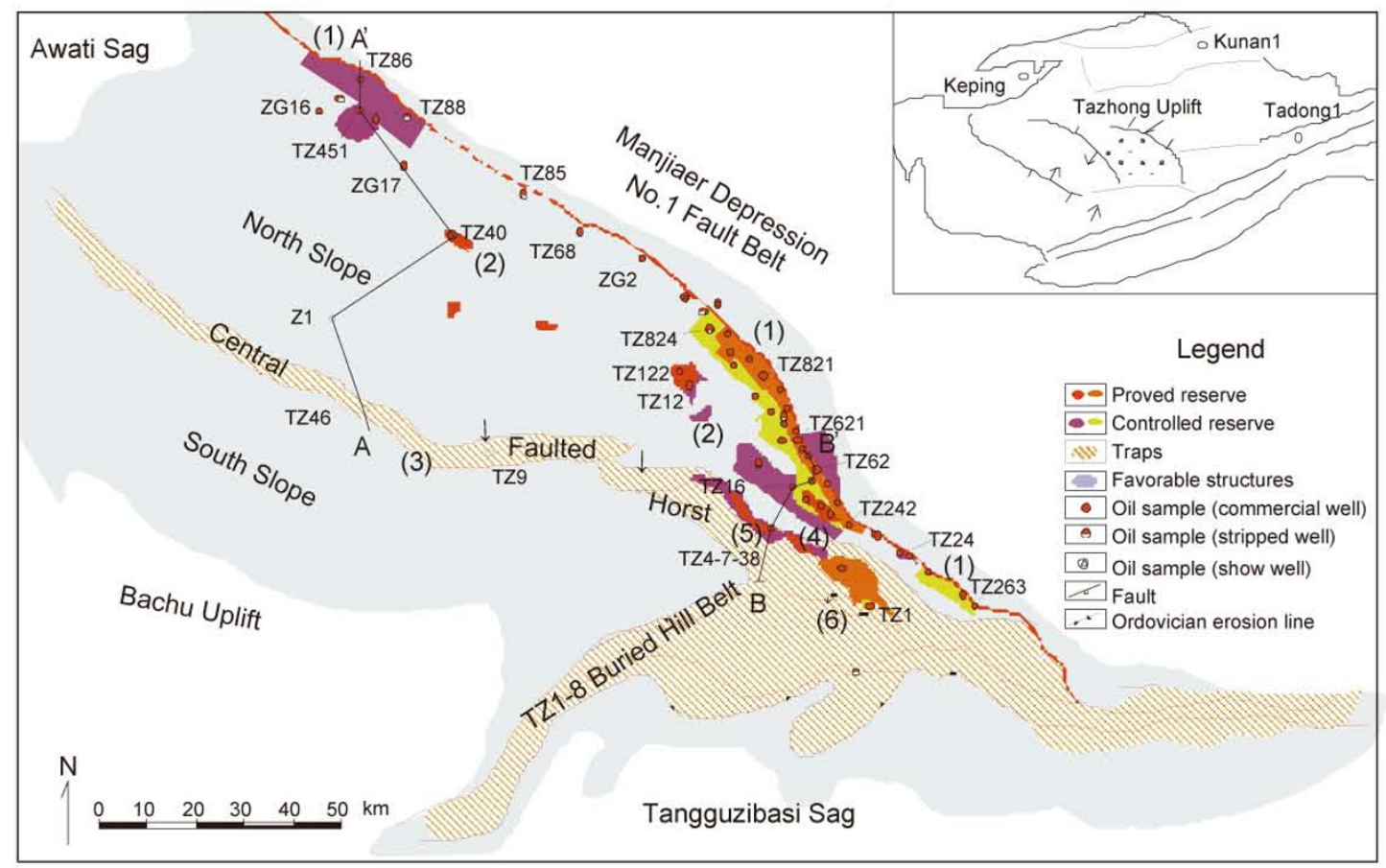

(a) Tectonic units of the Central Uplift and locations of oil samples collected

(1) No.1 Fault Belt; (2) TZ47-15 Block; (3) TZ2 Block; (4) TZ16 Block; (5) TZ4 Block; (6) TZ1-6 Block;
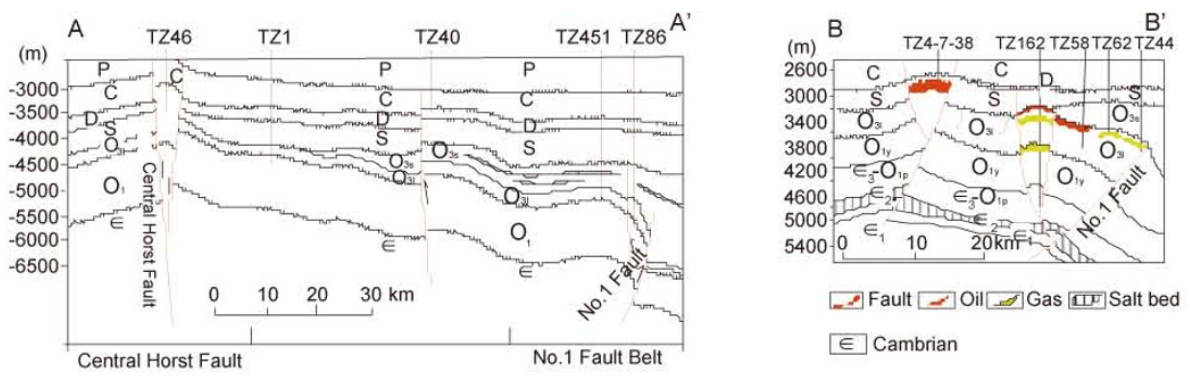

(b) Schematic structural cross section of the Central Uplift. See Fig. 1(a) for the map location of the cross section

Fig. 1 Structural diagram and oil distribution of the Tazhong Uplift

The evolution of the Tazhong Uplift includes five stages (Lü et al, 2004): 1) The stable platform development stage occurred in the early Caledonian when the Cambrian-early Ordovician sediments were deposited with a maximum depositional center in the east; 2) The isolated tableland development stage of the middle-late Caledonian (Middle Ordovician-Silurian) was marked by the establishment of the No.1 Fault (early to late Ordovician); 3) In the early 
Hercynian stage at the end of the Devonian, the SilurianDevonian sedimentary succession was extensively eroded in higher positions of the uplift, especially in the Central Faulted Horst Belt; 4) The late Hercynian stage (CarboniferousPermian) (Jia, 1999); 5) The Indosinian-Himalayan orogenic stage. The overall fluctuations of the uplift have had little impact on the Paleozoic structures since the Mesozoic.

Most of the oils in the Tazhong Uplift were discovered in the upper Ordovician Lianglitage Formation (dominant reservoir) in the No.1 Fault Belt (approximately $260 \mathrm{~km}$ in length) (Han et al, 2007; 2008), which is a large-scale thrust belt developed from the end of the early Ordovician to the beginning of the late Ordovician. The No.1 Fault developed in the belt was active during the Caledonian orogeny, cutting the Ordovician, Cambrian and upper Proterozoic (Sinian) strata, as well as the pre-Sinian basement (Fig. 1(b)). The fault was reactivated during subsequent orogenies. Faults and their associated fractures developed in the uplift have been considered to be the main pathways for upward hydrocarbons migration (Cai et al, 2001). The Carboniferous is the second most important oil-producing formation, with the hydrocarbons mostly accumulated in the TZ4 well block. However, only small amounts of oil have been discovered from Silurian reservoirs up to the present with the hydrocarbons mainly accumulated in the clastic rocks or their associated pools.

\section{Samples and experiment}

Fifty eight drill stem test (DST) oil samples from the Ordovician reservoirs and eight potential source rock samples from the Ordovician and Cambrian were collected (Fig. 1(a)). The details of the oil samples are given in Table 1. All the oil and source rock samples were analyzed for their molecular compositions, and 28 of them were selected for compound specific stable carbon isotope analysis of $n$-alkanes. Briefly, the oil and rock extracts were deasphalted before fractionation on a neutral alumina chromatographic column. The samples were separated into saturated hydrocarbons, aromatic hydrocarbons and polar fraction, by sequential elution with $n$-hexane, toluene and chloroform. For quantitative determination of aliphatic and aromatic hydrocarbons, absolute concentrations of known amounts of standard compounds $(20 \mathrm{R}-5 \alpha(\mathrm{H}) 14 \alpha(\mathrm{H}) 17 \alpha(\mathrm{H})$-d4-cholestane and $\mathrm{d} 8$-dibenzothiophene) were added to the concentrated extracts prior to the fractionation step. The details of the analytical procedures and instrument conditions are similar to those reported by Jiang et al (2001). The experimental procedures for carbonate fluid inclusion analysis are similar to those reported by Gong et al (2007). Briefly, the samples were disaggregated into 1-2 $\mathrm{mm}$ particles and then treated with organic solvents (methanol and dichloromethane) to remove any surface oils or contamination. The clean samples were then crushed under solvent (dichloromethane) to extract the inclusion oils. The concentrated inclusion oils were then prepared for immediate gas chromatography-mass spectrometry (GC-MS) analysis without group composition separation to avoid hydrocarbon loss.

\subsection{Gas chromatography-flame ionization detection and gas chromatography-mass spectrometry}

Gas chromatography-flame ionization detection (GCFID) of all oil samples was performed on a Carlo Erba 160 GC fitted with a PONA column $(60 \mathrm{~m}, 0.25 \mathrm{~mm}$ i.d., 0.32 $\mu \mathrm{m}$ film thickness). The GC oven was initially set at $35^{\circ} \mathrm{C}$ for $10 \mathrm{mins}$ and then programmed to $300^{\circ} \mathrm{C}$ at $4^{\circ} \mathrm{C} / \mathrm{min}$ with final holding time for 40 mins. Gas chromatography-mass spectrometric analyses (GC-MS) of saturated and aromatic hydrocarbons of the free oils and the whole fluid inclusion (FI) oils were carried out on a Hewlett-Packard 5890 GC coupled to a mass selective detector fitted with an HP-1 fused silica column (30 m, $0.25 \mathrm{~mm}$ i.d., $0.25 \mu \mathrm{m}$ film thickness). For analyzing saturated fractions, the $\mathrm{GC}$ oven was initially set at $50^{\circ} \mathrm{C}$ for 2 mins, programmed to $100^{\circ} \mathrm{C}$ at $2^{\circ} \mathrm{C} / \mathrm{min}$ and then to $310^{\circ} \mathrm{C}$ at $3^{\circ} \mathrm{C} / \mathrm{min}$, with a final holding time of $15 \mathrm{mins}$. For analyzing aromatic hydrocarbons, the $\mathrm{GC}$ oven was initially set at $60^{\circ} \mathrm{C}$, programmed to $150^{\circ} \mathrm{C}$ at $8^{\circ} \mathrm{C} / \mathrm{min}$ and then to $320^{\circ} \mathrm{C}$ at $4^{\circ} \mathrm{C} / \mathrm{min}$, with a final holding time of $10 \mathrm{mins}$. The mass spectrometer was operated in selected ion monitoring mode, targeting the molecular ions and characteristic fragment ions of selected aromatic hydrocarbons. Peak identifications and quantitative methods are reported by Jiang et al (2001).

\subsection{Gas chromatography-tandem mass spectrometry}

Gas chromatography-tandem mass spectrometry (GCMS-MS) analyses of saturated hydrocarbon fractions were performed on a VG hybrid 70 SQ equipped with an HP 5890 gas chromatograph and under the control of an Opus data system. The column used was a $35 \mathrm{~m} \times 0.32 \mathrm{~mm}$ i.d. fused silica high temperature capillary column coated with the DB-5 stationary phase. The samples were injected into a split/splitless injector in splitless mode, with an injector temperature of $320^{\circ} \mathrm{C}$. Helium was used as the carrier gas, with a constant flow rate of $1.5 \mathrm{~mL} / \mathrm{min}$. The oven temperature was initially set at $60^{\circ} \mathrm{C}$, then ramped to $320^{\circ} \mathrm{C}$ at $4^{\circ} \mathrm{C} / \mathrm{min}$ with a final holding time of 20 mins. For GC-MSMS analysis, the instrument was run in the parent-daughter mode, using nitrogen as a collision gas $\left(3 \times 10^{-3}\right.$ mbar, 20 $\mathrm{eV})$. The following transitions were monitored: 1) $\mathrm{m} / \mathrm{z} 358$, $372,386,400,414 \rightarrow 217$ for steranes; 2 ) $\mathrm{m} / \mathrm{z} 414 \rightarrow 231$ for dinosteranes; and 3) m/z 398, 412 $\rightarrow 191$ for hopanes.

\subsection{Compound specific stable carbon isotope analyses}

For compound specific stable carbon isotope analyses, $n$-alkanes in the crude oils were isolated from the saturated hydrocarbon fractions with $5 \AA$ molecular sieves but isolation of the $n$-alkanes in the inclusion oils was not possible due to the minute quantity of oil available. Compound specific isotope analyses (CSIA) were carried out on a Micromass IsoPrime mass spectrometer attached to an HP $6890 \mathrm{GC}$. A $60 \mathrm{~m} \times 0.25 \mathrm{~mm}$ i.d. capillary column coated with $0.25 \mu \mathrm{m}$ $5 \%$ phenyl-methyl-silicone stationary phase was fitted to the $\mathrm{GC}$. The $\mathrm{GC}$ oven was programmed from $50^{\circ} \mathrm{C}$ to $310^{\circ} \mathrm{C}$ at $3^{\circ} \mathrm{C} / \mathrm{min}$ with the initial and final holding time of 1 and 30 mins, respectively. The carrier gas used was helium at a flow 
Table 1 Origin and gross compositions of Tazhong oils and condensates

\begin{tabular}{|c|c|c|c|c|c|c|c|c|c|c|c|c|c|c|c|c|c|}
\hline $\begin{array}{c}\text { Tectonic } \\
\text { unit }\end{array}$ & $\begin{array}{l}\text { Lab. } \\
\text { No. }\end{array}$ & Well & Depth, m & Strata & $\begin{array}{c}\text { Sat } \\
\%\end{array}$ & $\begin{array}{c}\text { Aro } \\
\%\end{array}$ & $\begin{array}{c}\text { Resin } \\
\%\end{array}$ & $\begin{array}{c}\text { Asph } \\
\%\end{array}$ & $\begin{array}{l}\text { Sat/ } \\
\text { Aro }\end{array}$ & $\begin{array}{l}\mathrm{Pr} / \\
\mathrm{Ph}\end{array}$ & $\begin{array}{c}\mathrm{Pr} / \\
n \mathrm{C}_{17}\end{array}$ & $\begin{array}{l}\mathrm{Ph} / \\
n \mathrm{C}_{18}\end{array}$ & $\begin{array}{l}\left(n-\mathrm{C}_{21}+n-\mathrm{C}_{2}\right) / \\
\left(n-\mathrm{C}_{28}+n-\mathrm{C}_{29}\right)\end{array}$ & $\begin{array}{l}\sum n-C_{21}^{-1} \\
\sum n-C_{22}^{+}\end{array}$ & $\begin{array}{l}\text { Main } \\
\text { peak }\end{array}$ & PI-I & PI-II \\
\hline & 1 & TZ86 & $6273-6320$ & $\mathrm{O}_{3}$ & 83.4 & 12.6 & 2.6 & 1.3 & 6.6 & 0.97 & 0.34 & 0.42 & 3.56 & 8.23 & $n \mathrm{C}_{10}$ & 2.98 & 40.8 \\
\hline & 2 & TZ452 & $6376.76-6550$ & $\mathrm{O}_{1+2}$ & 73.4 & 17.9 & 5.2 & 3.5 & 4.1 & 1.34 & 0.25 & 0.21 & 4.82 & 5.99 & $n \mathrm{C}_{10}$ & 3.04 & 31.55 \\
\hline & 3 & ZG16 & $6230-6269$ & $\mathrm{O}$ & 91.6 & 7.6 & 0.9 & & 12.1 & 1.03 & 0.37 & 0.43 & 3.65 & 8.17 & $n \mathrm{C}_{10}$ & - & - \\
\hline & 4 & TZ451 & $6229.14-6297.62$ & $\mathrm{O}_{3}$ & 89.2 & 8.8 & 1.0 & 1 & 10.1 & 1.02 & 0.35 & 0.43 & 5.88 & 10.37 & $n \mathrm{C}_{8}$ & 3.19 & 40.55 \\
\hline & 5 & TZ45 & $6020-6150$ & $\mathrm{O}_{3}$ & 78.6 & 8.6 & 4.3 & 8.6 & 9.1 & 1.04 & 0.35 & 0.43 & 3.33 & 11.74 & $n \mathrm{C}_{10}$ & 2.98 & 40.41 \\
\hline & 6 & TZ88 & $6560-7260$ & $\mathrm{O}_{3}-\mathrm{O}_{1}$ & 49.7 & 25.8 & 18.4 & 6.1 & 1.9 & 1.05 & 0.48 & 0.56 & 5.03 & 8.87 & $n \mathrm{C}_{12}$ & 2.28 & 29.01 \\
\hline & 7 & ZG17 & $6438-6448$ & $\mathrm{O}$ & 94.3 & 5.5 & 0.2 & & 17.0 & 1.19 & 0.17 & 0.17 & 3.69 & 9.29 & $n \mathrm{C}_{9}$ & - & - \\
\hline & 8 & TZ85 & $6322-6415$ & $\mathrm{O}_{3}$ & 72.0 & 19.1 & 5.1 & 3.8 & 3.8 & 1.07 & 0.37 & 0.44 & 3.54 & 7.97 & $n \mathrm{C}_{8}$ & 2.45 & 38.83 \\
\hline & 9 & ZG2 & $5866-5893$ & $\mathrm{O}$ & 94.8 & 4.9 & 0.2 & & 19.2 & 1.12 & 0.27 & 0.29 & 3.71 & 9.91 & $n \mathrm{C}_{19}$ & - & - \\
\hline & 10 & TZ54 & $5832-5858$ & $\mathrm{O}_{3}$ & 82.9 & 8.6 & 4.6 & 3.9 & 9.6 & 0.97 & 0.3 & 0.37 & 3.13 & 6.93 & $n \mathrm{C}_{10}$ & 4.25 & 38.56 \\
\hline & 11 & TZ826 & $5668-5672$ & $\mathrm{O}_{3}$ & 80.2 & 7.0 & 10.5 & 2.3 & 11.5 & 1.2 & 0.29 & 0.29 & 3.83 & 6.2 & $n \mathrm{C}_{12}$ & 5.55 & 33.33 \\
\hline & 12 & TZ824 & $5613-5621$ & $\mathrm{O}_{3}$ & 83.0 & 11.8 & 5.2 & 0 & 7.0 & 0.95 & 0.34 & 0.41 & 2.95 & 4.26 & $n \mathrm{C}_{12}$ & 2.47 & 34.73 \\
\hline & 13 & TZ828 & $5595-5603$ & $\mathrm{O}_{3}$ & 75.8 & 11.1 & 13.1 & 0 & 6.8 & 0.99 & 0.27 & 0.33 & 3.25 & 6.73 & $n \mathrm{C}_{10}$ & 3.28 & 37.83 \\
\hline & 14 & TZ82 & $5349.52-5385$ & $\mathrm{O}_{3}$ & 85.4 & 11.2 & 1.1 & 2.2 & 7.6 & 0.99 & 0.27 & 0.35 & 4.66 & 11.81 & $n \mathrm{C}_{10}$ & 3.30 & 38.81 \\
\hline & 15 & TZ82 & $5430-5487$ & $\mathrm{O}_{3}$ & 84.6 & 11.0 & 2.2 & 2.2 & 7.7 & 1 & 0.29 & 0.37 & 5.34 & 13.62 & $n \mathrm{C}_{10}$ & 3.44 & 40.57 \\
\hline & 16 & TZ825 & $5225.42-5300$ & $\mathrm{O}_{3}$ & 83.8 & 12.1 & 1.0 & 3 & 6.9 & 1.19 & 0.3 & 0.3 & 4.58 & 6.42 & $n \mathrm{C}_{11}$ & 3.42 & 40.55 \\
\hline & 17 & TZ823 & $5369-5490$ & $\mathrm{O}_{3}$ & 90.7 & 7.9 & 1.4 & 0 & 11.5 & 1.23 & 0.26 & 0.23 & 2.32 & 3.01 & $n \mathrm{C}_{12}$ & 2.96 & 35.70 \\
\hline & 18 & TZ821 & $5212.64-5250.2$ & $\mathrm{O}_{3}$ & 87.1 & 10.8 & 2.2 & 0 & 8.1 & 1.35 & 0.27 & 0.22 & 2.87 & 4.21 & $n \mathrm{C}_{10}$ & 3.45 & 40.81 \\
\hline & 19 & TZ83 & $5411-5433$ & $\mathrm{O}_{3}$ & 87.3 & 11.0 & 1.7 & 0 & 7.9 & 0.99 & 0.32 & 0.40 & 2.92 & 5.62 & $n \mathrm{C}_{12}$ & 4.75 & 37.12 \\
\hline & 20 & TZ83 & $5666.1-5686.7$ & $\mathrm{O}_{1}$ & 88.3 & 10.4 & 1.3 & 0 & 8.5 & 1.04 & 0.25 & 0.28 & 2.27 & 5.05 & $n \mathrm{C}_{10}$ & 4.25 & 39.91 \\
\hline & 21 & TZ721 & $4911.37-4980.4$ & $\mathrm{O}_{3}$ & 82.9 & 7.5 & 9.6 & 0 & 11.1 & 1.3 & 0.26 & 0.24 & 4.43 & 7.59 & $n \mathrm{C}_{12}$ & 3.22 & 29.33 \\
\hline \multirow{23}{*}{$\begin{array}{l}\text { No.1 } \\
\text { Fault } \\
\text { Belt }\end{array}$} & 22 & TZ721 & $5030-5070$ & $\mathrm{O}_{3}$ & 47.3 & 26.1 & 9.2 & 17.4 & 1.8 & 0.83 & 0.4 & 0.58 & 2.96 & 6.48 & $n \mathrm{C}_{8}$ & 2.35 & 35.04 \\
\hline & 23 & TZ721 & $5355.5-5505$ & $\mathrm{O}_{1}$ & 87.2 & 11.7 & 1.1 & 0 & 7.5 & 0.96 & 0.25 & 0.27 & 1.58 & 2.08 & $n \mathrm{C}_{12}$ & 3.67 & 40.32 \\
\hline & 24 & TZ62-3 & $5072.46-5165$ & $\mathrm{O}_{3}$ & 77.8 & 16.7 & 3.2 & 2.4 & 4.7 & 1.3 & 0.26 & 0.23 & 3.73 & 6.3 & $n \mathrm{C}_{12}$ & 3.16 & 33.90 \\
\hline & 25 & TZ622 & 4913.52-4925 & $\mathrm{O}_{3}$ & 78.3 & 10.6 & 8.2 & 2.9 & 7.4 & 0.98 & 0.27 & 0.3 & 1.8 & 2.21 & $n \mathrm{C}_{17}$ & 2.78 & 35.26 \\
\hline & 26 & TZ30 & $4997-5026$ & $\mathrm{O}_{3}$ & 80.9 & 14.0 & 3.9 & 1.1 & 5.8 & 0.93 & 0.25 & 0.31 & 3.35 & 4.42 & $n \mathrm{C}_{13}$ & 4.78 & 27.17 \\
\hline & 27 & TZ72 & $5125-5130$ & $\mathrm{O}_{3}$ & 73.5 & 22.7 & 3.8 & 0 & 3.2 & 0.99 & 0.23 & 0.28 & 3.39 & 5.94 & $n \mathrm{C}_{11}$ & 4.79 & 39.77 \\
\hline & 28 & TZ62-1 & $4892.07-4973.76$ & $\mathrm{O}_{3}$ & 75.6 & 17.9 & 4.5 & 1.9 & 4.2 & 0.88 & & 0.28 & 3.26 & 2.56 & $n \mathrm{C}_{19}$ & 3.59 & 32.39 \\
\hline & 29 & TZ722 & $5356.7-5750$ & $\mathrm{O}$ & 94.2 & 5.6 & 0.3 & & 16.9 & 1.24 & 0.24 & 0.22 & 6.16 & 6.14 & $n \mathrm{C}_{12}$ & - & - \\
\hline & 30 & TZ621 & $4851.1-4885$ & $\mathrm{O}_{3}$ & 63.1 & 15.6 & 7.5 & 13.8 & 4.0 & 1.49 & 0.18 & 0.13 & 3.52 & 3.84 & $n \mathrm{C}_{13}$ & 3.03 & 29.26 \\
\hline & 31 & TZ62-2 & $4773.53-4825$ & $\mathrm{O}_{3}$ & 86.4 & 11.4 & 2.3 & 0 & 7.6 & 1.16 & 0.24 & 0.25 & 6.07 & 9.07 & $n \mathrm{C}_{11}$ & 3.26 & 39.21 \\
\hline & 32 & TZ44 & $4822-4832$ & $\mathrm{O}_{3}$ & 83.6 & 11.0 & 1.4 & 4.1 & 7.6 & 1.16 & 0.26 & 0.32 & - & 51.96 & $n \mathrm{C}_{11}$ & 3.89 & 41.43 \\
\hline & 33 & TZ73 & $4761-4775$ & $\mathrm{O}_{3}$ & 77.6 & 16.4 & 3.3 & 2.6 & 4.7 & 1.32 & 0.3 & 0.31 & 3.41 & 13.35 & $n \mathrm{C}_{10}$ & 4.79 & 37.51 \\
\hline & 34 & TZ62 & $4700.5-4758$ & $\mathrm{O}_{3}$ & 63.5 & 10.0 & 25.1 & 1.4 & 6.4 & - & - & - & - & - & - & - & - \\
\hline & 35 & TZ623 & $4809-4815$ & $\mathrm{O}_{3}$ & 91.3 & 7.2 & 1.5 & 0 & 12.7 & 1.23 & 0.25 & 0.24 & 6.84 & 6.85 & $n \mathrm{C}_{15}$ & 3.29 & 29.52 \\
\hline & 36 & TZ70C & $4754-4830$ & $\mathrm{O}_{3}$ & 83.5 & 12.1 & 4.4 & 0 & 6.9 & 1.31 & 0.26 & 0.24 & 6.16 & 11.35 & $n \mathrm{C}_{11}$ & 3.43 & 42.39 \\
\hline & 37 & TZ242 & $4515.56-4546.56$ & $\mathrm{O}_{3}$ & 83.8 & 15.2 & 1.0 & 0 & 5.5 & 1.3 & 0.24 & 0.23 & 16.83 & 12.99 & $n \mathrm{C}_{14}$ & 3.81 & 31.84 \\
\hline & 38 & TZ241 & $4618.47-4725.74$ & $\mathrm{O}_{3}$ & 90.4 & 8.8 & 0.8 & 0 & 10.3 & 0.98 & 0.23 & 0.28 & 4.93 & 7.01 & $n \mathrm{C}_{13}$ & 5.17 & 34.42 \\
\hline & 39 & TZ244 & $4407-4433.64$ & $\mathrm{O}_{3}$ & - & - & - & - & - & 1.19 & 0.25 & 0.31 & - & 65.26 & $n \mathrm{C}_{11}$ & 2.68 & 35.61 \\
\hline & 40 & TZ243 & $4387.03-4547.9$ & $\mathrm{O}_{3}$ & 78.8 & 18.8 & 2.4 & 0 & 4.2 & 1.37 & 0.23 & 0.22 & 17.92 & 19.95 & $n \mathrm{C}_{12}$ & 2.65 & 36.00 \\
\hline & 41 & TZ24 & $4461.1-4483.48$ & $\mathrm{O}_{3}$ & 89.7 & 7.2 & 3.1 & 0 & 12.5 & 1.25 & 0.23 & 0.24 & 6.44 & 16.93 & $n \mathrm{C}_{11}$ & 5.22 & 20.98 \\
\hline & 42 & TZ261 & $4357-4380$ & $\mathrm{O}_{3}$ & 90.4 & 6.7 & 2.9 & 0 & 13.5 & 1.25 & 0.23 & 0.21 & 12.52 & 10.7 & $n \mathrm{C}_{12}$ & 2.44 & 41.51 \\
\hline & 43 & TZ26 & $4300-4360$ & $\mathrm{O}_{3}$ & 87.1 & 9.7 & 3.2 & 0 & 9.0 & 1.4 & 0.29 & 0.27 & 8 & 13.32 & $n \mathrm{C}_{12}$ & 2.58 & 34.2 \\
\hline & 44 & TZ263 & $4310-4342$ & $\mathrm{O}_{3}$ & 87.2 & 9.6 & 3.2 & 0 & 9.1 & 1.02 & 0.29 & 0.36 & 15.17 & 15.69 & $n \mathrm{C}_{12}$ & 2.13 & 34.00 \\
\hline
\end{tabular}




\begin{tabular}{|c|c|c|c|c|c|c|c|c|c|c|c|c|c|c|c|c|}
\hline \multirow{3}{*}{ TZ47-15 } & 45 & TZ122 & $4707.07-4733.92$ & $\mathrm{O}_{3}$ & 70.4 & 20.4 & 6.8 & 2.5 & 3.5 & 0.88 & 0.29 & 0.37 & 1.61 & 2.12 & $n \mathrm{C}_{13}$ & 4.1030 .36 \\
\hline & 46 & $\mathrm{TZ12}$ & $4695.5-4777.5$ & $\mathrm{O}_{3}$ & 47.9 & 19.9 & 14.4 & 17.8 & 2.4 & 0.85 & 0.29 & 0.41 & 3.21 & 5.58 & $n \mathrm{C}_{10}$ & 3.6630 .32 \\
\hline & 47 & TZ15 & $4656-4673$ & $\mathrm{O}_{3}$ & 33.5 & 34.4 & 10.5 & 21.5 & 1.0 & 1.05 & 0.45 & 0.62 & - & 7.35 & $n \mathrm{C}_{15}$ & 3.8618 .31 \\
\hline \multirow{5}{*}{ TZ16 } & 48 & $\mathrm{TZ162}$ & $5048-5070$ & $\mathrm{O}_{1 \mathrm{y}}$ & 73.1 & 19.4 & 7.4 & 0 & 3.8 & 1.15 & 0.22 & 0.25 & 3.28 & 10.16 & $\mathrm{nC}_{11}$ & 4.2232 .21 \\
\hline & 49 & TZ16 & $4244.6-4259.5$ & $\mathrm{O}_{3}$ & 62.5 & 16.7 & 12.0 & 8.8 & 3.7 & 1.24 & 0.3 & 0.29 & 3.97 & 7.65 & $n \mathrm{C}_{12}$ & 4.0634 .17 \\
\hline & 50 & TZ168 & 4374-4399 & $\mathrm{O}_{3}$ & 68.0 & 22.7 & 5.3 & 4 & 3.0 & 1.25 & 0.26 & 0.26 & 4.96 & 8.17 & $n \mathrm{C}_{14}$ & 3.4835 .68 \\
\hline & 51 & $\mathrm{TZ168}$ & $4670-4686$ & $\mathrm{O}_{3}$ & 59.0 & 25.2 & 7.9 & 7.9 & 2.3 & 1.4 & 0.27 & 0.25 & 6.02 & 11.19 & $n \mathrm{C}_{13}$ & $3.85 \quad 38.24$ \\
\hline & 52 & TZ161 & 4289-4306 & $\mathrm{O}_{3}$ & 62.7 & 23.9 & 8.2 & 5.2 & 2.6 & 0.94 & 0.25 & 0.34 & 6.47 & 11.82 & $n \mathrm{C}_{14}$ & 3.6035 .91 \\
\hline \multirow{3}{*}{ TZ1-6 } & 53 & TZ47-38 & $3936.43-3985.5$ & $\mathrm{O}_{1 \mathrm{p}}$ & 29.9 & 36.3 & 12.5 & 21.4 & 0.8 & 0.77 & 0.34 & 0.62 & 3.01 & 5.92 & $n \mathrm{C}_{13}$ & 3.4421 .67 \\
\hline & 54 & TZ6 & $4248.5-4265$ & $\mathrm{O}$ & 43.7 & 40.9 & 7.4 & 8.7 & 1.1 & 0.97 & 0.31 & 0.39 & 5.80 & 9.25 & $n \mathrm{C}_{12}$ & $6.31 \quad 19.50$ \\
\hline & 55 & TZ52 & 3811-3819 & $\mathrm{O}_{1 \mathrm{y}}$ & 79.6 & 16.3 & 4.1 & 0 & 4.9 & 1.83 & 0.26 & 0.19 & 4.63 & 12.17 & $n \mathrm{C}_{9}$ & 2.8945 .04 \\
\hline \multirow{3}{*}{$\begin{array}{c}\text { End } \\
\text { member } \\
\text { oil }\end{array}$} & 56 & TZ62 & $4052.98-4073.58$ & $\mathrm{~S}$ & 47.8 & 16.4 & 29.0 & 6.8 & 2.9 & 1.14 & 0.3 & 0.31 & 5.49 & 7.71 & $n \mathrm{C}_{12}$ & 3.4538 .76 \\
\hline & 57 & YM2 & $5940-5953$ & $\mathrm{O}_{1}$ & 50.9 & 27.2 & 11.6 & 10.4 & 1.9 & - & - & - & - & - & - & - \\
\hline & 58 & TD2 & $4561.93-5040$ & $\mathrm{O}_{1}-\in$ & 45 & 26 & 13 & 16 & 1.7 & - & - & - & - & - & - & - \\
\hline
\end{tabular}

rate of $1 \mathrm{~mL} / \mathrm{min}$ with the injector operating at constant flow rate.

The stable carbon isotopic compositions $\left(\delta^{13} \mathrm{C}\right)$ were calculated by the integration of the masses 44,45 and 46 ion current counts of the $\mathrm{CO}_{2}$ peaks produced by the combustion (copper oxide reaction furnace at $850^{\circ} \mathrm{C}$ ) of hydrocarbons separated by gas chromatography. $\mathrm{A} \mathrm{CO}_{2}$ reference gas (calibrated to Vienna Peedee belemnite) with a known $\delta^{13} \mathrm{C}$ was pulsed into the mass spectrometer and the isotopic composition of samples was reported in the $\delta$ notation relative to the reference gas. The average values of each sample for at least two runs were reported and only the results with a standard deviation of less than $0.3 \%$ were used.

\section{Results and discussion}

\subsection{Mixed oil geochemistry}

\subsubsection{Physical properties and group compositions}

The Ordovician oils in the Tazhong Uplift have widely variable bulk characteristics, and range from colourless or yellowish condensates, dark brown oils, and white waxy to heavy oils. Most of the hydrocarbons were discovered primarily from the Middle-Upper Ordovician strata in the No.1 Fault Belt. Only a few wells produced commercial oil from the Carboniferous and Silurian in the No.1 Fault Belt owing to small faults cutting through the underlying thick Santamu Formation within this belt (Fig. 1). Most of the Ordovician oils, especially those in the No.1 Fault Belt, are characterized by low density $\left(0.76-0.99 \mathrm{~g} / \mathrm{cm}^{3}\right.$, with an average of $\left.0.83 \mathrm{~g} / \mathrm{cm}^{3}\right)$, low sulfur content $(0.02 \%$ $1.71 \%$, with an average of $0.25 \%$ ) and low-moderate wax content $(0.34 \%-16.20 \%$, with an average of $7.13 \%)$ (Fig. 2). The Lower Ordovician oils generally have similar physical properties to the $\mathrm{O}_{2+3}$ oils except the oils from TZ4-7-38 $\left(\mathrm{O}_{1 \mathrm{p}}\right)$, TZ83 and TZ721 wells. The TZ4-7-38 oil has an extremely high level of $\mathrm{H}_{2} \mathrm{~S}(2.59 \%)$ in its associated gas, which has been considered to be the product of in-reservoir thermochemical sulfate reduction (TSR) (Cai et al, 2004; Chen et al, 2008; Zhang et al, 2008).

The composition of liquid crude oil in the Tazhong Uplift coincides with the physical properties of the crude oil. The Ordovician oils have prominent saturated hydrocarbons
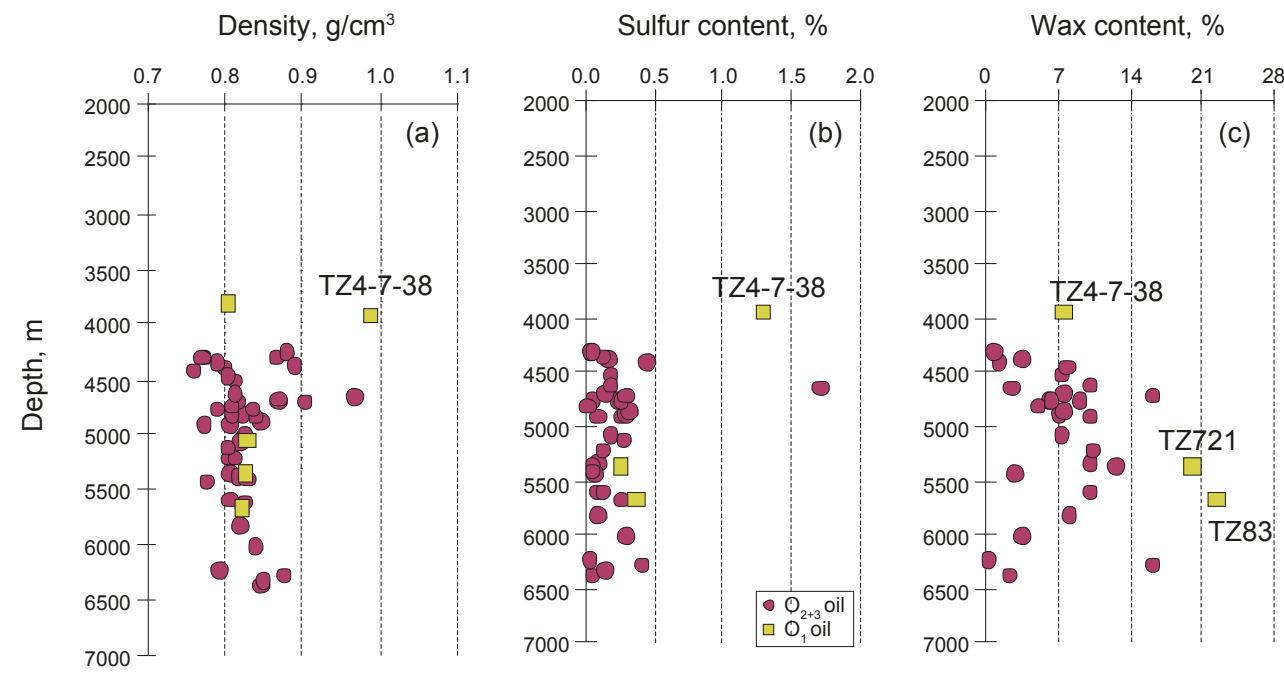

Fig. 2 Physical properties of the Ordovician oils in the Tazhong Uplift 
(29.9\%-94.8\%, with an average of $75.2 \%)$, with relatively low percentages of aromatic hydrocarbons $(4.9 \%-40.9 \%$, with an average of $15.1 \%)$ and resins + asphaltenes $(0.20 \%$ $35.8 \%$, with an average of $9.7 \%$ ) (Table 1). The ratios of total saturated to aromatic hydrocarbons of the Ordovician oils are within the range of 0.8-19.2 (Table 1). The physical properties and group compositions of the Ordovician oils suggest moderate-high thermal maturity of the oils.

\subsubsection{Chemical composition and distribution}

The Ordovician oils show a large variation of chemical composition and distribution, which change with the structural units where the oils are situated. The oils in the No.1 Fault Belt have a much higher concentration of $n$-alkanes $(10.3-207.1 \mu \mathrm{g} / \mathrm{mg}$ oil, average $85.6 \mu \mathrm{g} / \mathrm{mg}$ oil) and isoprenoids (2.6-18.0 $\mu \mathrm{g} / \mathrm{mg}$ oil, average $10.1 \mu \mathrm{g} / \mathrm{mg}$ oil) compared to the TZ47-15 tectonic belt $(36.3 \mu \mathrm{g} / \mathrm{mg}$ oil and 3.8 $\mu \mathrm{g} / \mathrm{mg}$ oil on average, respectively) (Table 2 ), corresponding to the high percentage of the saturated HCs observed in the Ordovician oils (Table 1). Most of the oils in the No.1 Fault Belt illustrate a unimodal distribution of $n$-alkanes enriched in low molecular weight with the main peak around $\mathrm{C}_{10-12}$ (Table 1). The ratios of $\left(n-\mathrm{C}_{21}+n-\mathrm{C}_{22}\right) /\left(n-\mathrm{C}_{28}+n-\mathrm{C}_{29}\right)$ and $\sum n \mathrm{C}_{21}{ }^{1} \sum n-\mathrm{C}_{22}{ }^{+}$of the Ordovician oils are as high as 1.58-17.92 (5.04 in average) and 2.08-65.26 (10.10 in average) (Table $1)$, respectively. A generally increasing trend of $\mathrm{Pr} / n-\mathrm{C}_{17}$ and $\mathrm{Ph} / n-\mathrm{C}_{18}$ ratios with increasing depth is observed for the Ordovician oils in the No.1 Fault Belt (Table 1). Thermal maturity and migration fractionation may have an impact on the ratios (Pang et al, 2003). The former is likely predominant since no clear migration fractionation based on nitrogen compounds is observed among the oils. The oils in the No.1 Fault Belt are also characterized by low abundance of steranes and hopanes (Table 2), which usually decrease with increasing maturity ( $\mathrm{Li}$ et al, 2003; 2005; Pang et al, 2003). This trend suggests that the oil was generated at relatively high thermal maturity.

Most Ordovician oils in the Tazhong Uplift display a "Vshaped" $\mathrm{C}_{27}, \mathrm{C}_{28}$ and $\mathrm{C}_{29}$ regular sterane relative abundances (Fig. 3). Only a small number of oil samples presented a linear or anti-" $\mathrm{L}$ " shape of steranes, including oils from the TZ452 $\left(\mathrm{O}_{1+2}\right)$, TZ1 $(\in, 3,755-3,768.5 \mathrm{~m}), \mathrm{TZ2} 2\left(\mathrm{C}_{\mathrm{III}}, \mathrm{O}_{3}\right)$ and TZ6 $\left(\mathrm{C}_{\mathrm{II}}, \mathrm{O}\right)$ wells (Fig. 3). This kind of oil usually displays relatively low concentrations of diasteranes, $\mathrm{C}_{21}$ and $\mathrm{C}_{22}$ pregnane series, as well as tricyclic terpane, and relatively high abundances of gammacerane (Fig. 3, Table 2), which were indicative of $\in-\mathrm{O}_{1}$ genetic affinity (Zhang et al, 2000a; Xiao et al, 2004; 2005).

$\mathrm{C}_{30}$ steranes, including 4-methyl sterane and dinosteranes, are detected in five out of the eight Ordovician oil samples analyzed by GC-MS-MS in this study (Fig. 4). The 24norcholestanes are also detected in several oil samples, which are used for identifying the oil sources (Zhang et al, 2000a; 2000b). Compared with the other crude oils, severe inhomogeneity was observed in oils from carbonate reservoirs along the No.1 Fault Belt, even between oils located within a very short distance (Fig. 3, Table 2), which suggest different oil sources and/or multiple oil charging episodes and strong heterogeneity of carbonate reservoirs. It is frequently observed that the pressure coefficient of the Ordovician carbonate reservoirs is quite different, as is the formation water (Zeng et

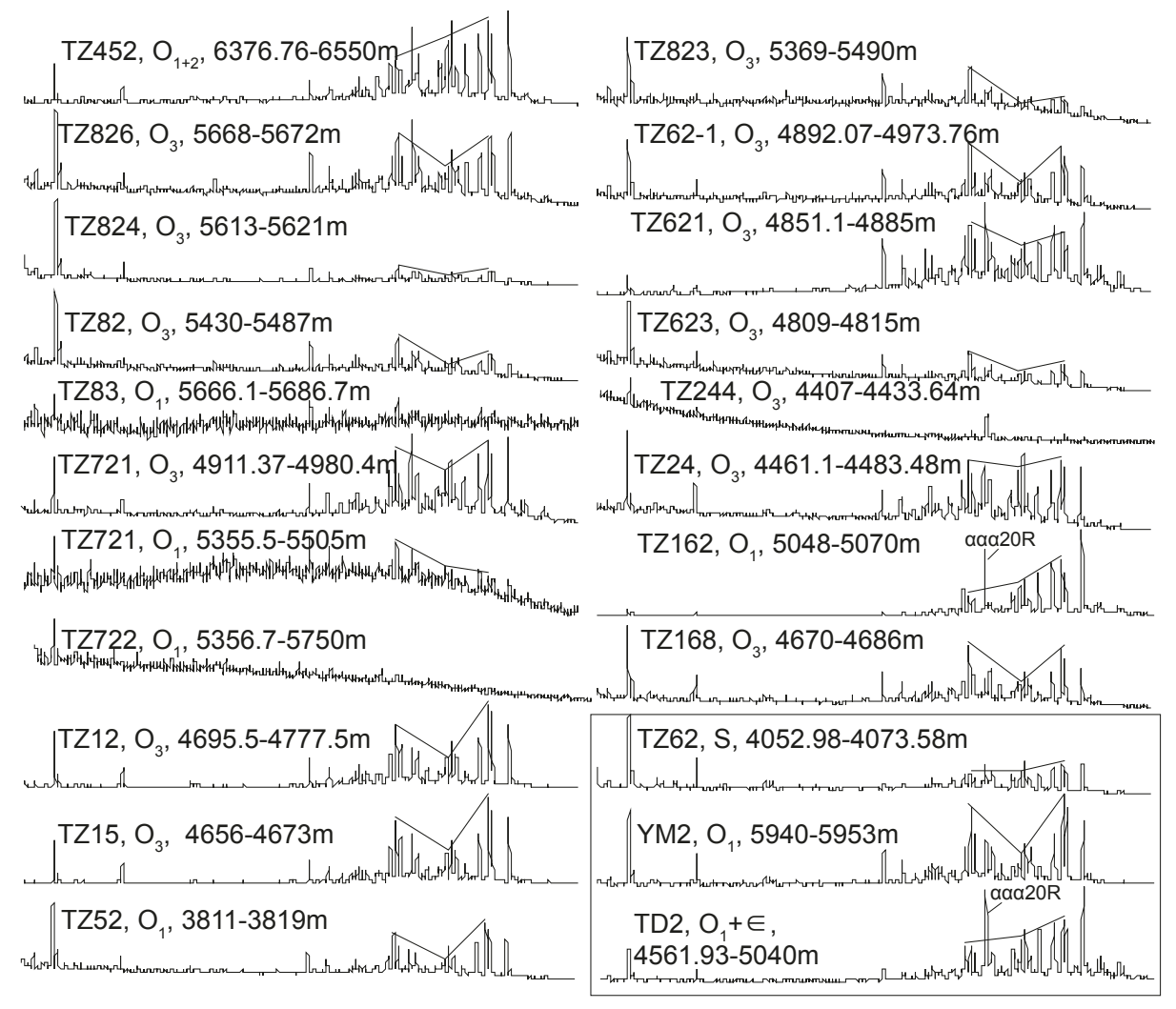

Fig. $3 \mathrm{M} / \mathrm{z} 217$ mass fragmentograms of saturated hydrocarbon fractions from selected oils in the Tazhong Uplift and the peripheral area showing representative sterane distributions 
Table 2 Basic geochemical parameters of the oils in the Tazhong Uplift

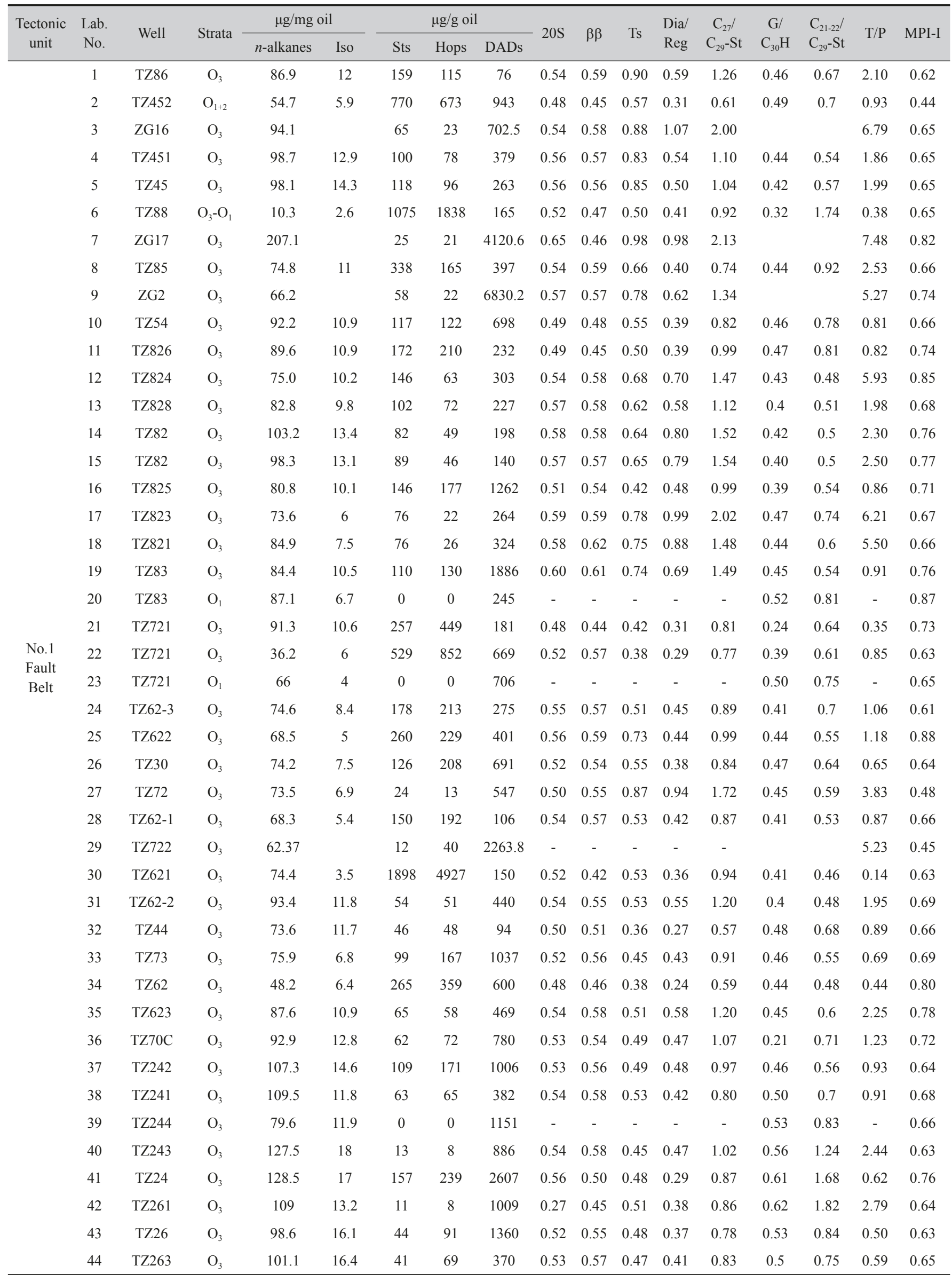




\begin{tabular}{|c|c|c|c|c|c|c|c|c|c|c|c|c|c|c|c|c|c|}
\hline \multirow{3}{*}{ TZ47-15 } & 45 & TZ122 & $\mathrm{O}_{3}$ & 62.9 & 5.2 & 177 & 469 & 113 & 0.51 & 0.57 & 0.50 & 0.38 & 0.94 & 0.44 & 0.81 & 0.37 & 0.74 \\
\hline & 46 & $\mathrm{TZ12}$ & $\mathrm{O}_{3}$ & 39.4 & 5 & 393 & 671 & 88 & 0.54 & 0.54 & 0.39 & 0.31 & 0.68 & 0.41 & 0.55 & 0.59 & 0.82 \\
\hline & 47 & TZ15 & $\mathrm{O}_{3}$ & 6.6 & 1.3 & 505 & 735 & 155 & 0.52 & 0.53 & 0.37 & 0.28 & 0.71 & 0.41 & 0.36 & 0.62 & 0.64 \\
\hline \multirow{5}{*}{ TZ16 } & 48 & TZ162 & $\mathrm{O}_{1 \mathrm{y}}$ & 26 & 2.1 & 336 & 561 & 5889 & 0.45 & 0.38 & 0.56 & 0.09 & 0.50 & 0.32 & 0.03 & 0.11 & 0.79 \\
\hline & 49 & TZ16 & $\mathrm{O}_{3}$ & 46.2 & 6.2 & 519 & 564 & 465 & 0.53 & 0.51 & 0.38 & 0.25 & 0.34 & 0.22 & 0.16 & 0.49 & 0.87 \\
\hline & 50 & TZ168 & $\mathrm{O}_{3}$ & 54.7 & 8.3 & 198 & 301 & 328 & 0.52 & 0.56 & 0.40 & 0.50 & 0.97 & 0.12 & 0.50 & 0.74 & 0.82 \\
\hline & 51 & TZ168 & $\mathrm{O}_{3}$ & 40.7 & 5.6 & 115 & 186 & 190 & 0.54 & 0.58 & 0.42 & 0.45 & 0.87 & 0.12 & 0.51 & 0.74 & 0.88 \\
\hline & 52 & TZ161 & $\mathrm{O}_{3}$ & 42.1 & 5.7 & 116 & 179 & 15 & 0.54 & 0.57 & 0.45 & 0.52 & 0.96 & 0.12 & 0.45 & 0.71 & 0.86 \\
\hline \multirow{3}{*}{ TZ1-6 } & 53 & TZ47-38 & $\mathrm{O}_{1 \mathrm{p}}$ & 7.0 & 0.9 & 226 & 225 & 217 & 0.53 & 0.56 & 0.44 & 0.49 & 1.00 & 0.20 & 0.92 & 1.12 & 0.79 \\
\hline & 54 & TZ6 & $\mathrm{O}$ & 31.4 & 3.8 & 172.8 & 210 & 11 & 0.52 & 0.55 & 0.41 & 0.45 & 0.78 & 0.18 & 0.50 & 0.77 & 0.96 \\
\hline & 55 & $\mathrm{TZ52}$ & $\mathrm{O}_{1 \mathrm{y}}$ & 82.7 & 8.3 & 142 & 320 & 241 & 0.54 & 0.52 & 0.38 & 0.29 & 0.73 & 0.09 & 0.43 & 0.40 & 0.9 \\
\hline \multirow{3}{*}{$\begin{array}{c}\text { End } \\
\text { member } \\
\text { oil }\end{array}$} & 56 & TZ62 & $\mathrm{S}$ & 31.7 & 6.5 & 403 & 441 & 183 & 0.54 & 0.45 & 0.37 & 0.22 & 0.64 & 0.38 & 0.5 & 1.26 & 1.25 \\
\hline & 57 & YM2 & $\mathrm{O}_{1}$ & 39.7 & 5.9 & 429 & 784 & 0 & 0.51 & 0.56 & 0.35 & 0.30 & 0.75 & 0.12 & 0.34 & 0.51 & 0.98 \\
\hline & 58 & TD2 & $\mathrm{O}_{1^{-}} \in$ & 0.2 & - & 6 & 23 & 0 & 0.46 & 0.40 & 0.36 & 0.12 & 0.51 & 0.40 & 0.13 & 0.15 & 0.85 \\
\hline
\end{tabular}

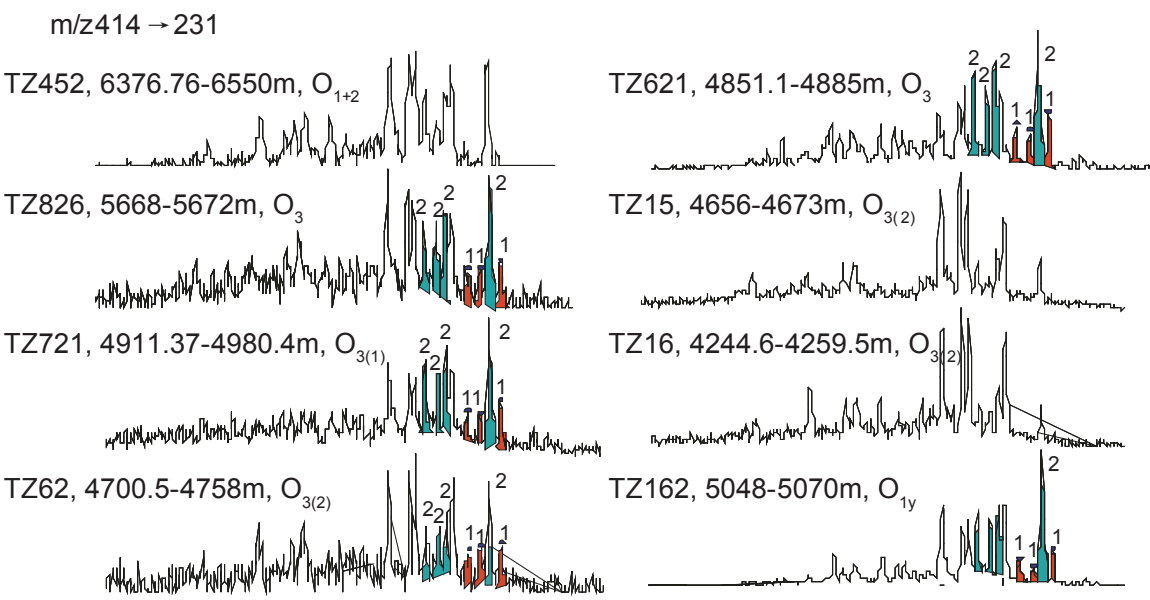

Fig. $4 \mathrm{M} / \mathrm{z} 414 \rightarrow 231$ parent-daughter ion chromatograms showing the methylsterane distributions in the Tazhong Uplift (1: $4 \alpha, 23,24$-methyl sterane $\left(\mathrm{C}_{30}\right) ; 2: 4 \alpha$-methyl-24-ethyl sterane $\left.\left(\mathrm{C}_{30}\right)\right)$

al, 2008; Peng, 2009 (unpublished data)).

The Ordovician oils in the TZ47-15 block are different in significantly higher abundances of steranes $(173-505 \mu \mathrm{g} / \mathrm{g}$ oil, an average of $358.3 \mu \mathrm{g} / \mathrm{g}$ oil) and hopanes $(469-735 \mu \mathrm{g} / \mathrm{g}$ oil, an average of $625 \mu \mathrm{g} / \mathrm{g}$ oil) (Table 2) compared with the other oils, e.g., the oils in the No.1 Fault Belt $(188.3 \mu \mathrm{g} / \mathrm{g}$ oil and $283.6 \mu \mathrm{g} / \mathrm{g}$ oil in average) and TZ1-6 Tectonic Belt (180.2 $\mu \mathrm{g} / \mathrm{g}$ oil and $251.7 \mu \mathrm{g} / \mathrm{g}$ oil in average) (Table 2), suggesting a relatively low thermal maturity as maturity parameters of diamondoids indicated. Apparent homogeneity was also observed for the oils in the TZ47-15 (Fig. 3, Table 2), which shows a very close genetic affinity among the crude oils.

The ratios of $20 \mathrm{~S} /(\mathrm{S}+\mathrm{R})$ and $\alpha \beta \beta /(\alpha \alpha \alpha+\alpha \beta \beta)$ for the $\mathrm{C}_{29}$ steranes in the oils from the Tazhong Uplift are primarily within the range of $0.5-0.55$ (the equilibrium point is about $0.52-0.55$ ) and $0.5-0.6$ (the equilibrium point is $0.67-0.71$ ) (Table 2), respectively. Most of the biomarker maturity parameters of the steranes and hopanes may have lost their effectiveness as maturity indicators. For example, some of the Ordovician oils in the No.1 Fault Belt are devoid of steranes and hopanes (Fig. 3), implying thermal cracking of the biomarkers. Diamondoids $(788.5 \mu \mathrm{g} / \mathrm{g}$ oil in average) were detected in almost all oil samples (Table 2), which have a high thermal stability and strong resistance of biodegradation compared with the other crude oil compounds (Chen et al, 1996; Zhang et al, 2005; Wei et al, 2007). High heptane values (Table 1) also indicate higher maturity levels of the Ordovician oils.

\subsection{Oil-source rock correlation}

\subsubsection{Correlation of biomarkers}

It has been pointed out that the middle-upper strata should be the main source rocks for the oils in the Tarim Basin (Zhang et al, 2000a), and this information is based on age diagnostic biomarkers, including 24-isopropylcholestanes, dinosteranes (4a, 23, 24-trimethylcholestanes), triaromatic dinosteroids, and 24-norcholestanes (Zhang et al, 2000a). After detailed study, Zhang et al $(2000 \mathrm{a} ; 2000 \mathrm{~b})$ identified that almost all Cambrian strata had linear or anti "L" shape distribution of $\mathrm{C}_{27}, \mathrm{C}_{28}$ and $\mathrm{C}_{29}$ steranes in rock extracts, whereas most of the middle-upper interval displayed "V" shapes. The Cambrian strata were characterized by relatively high 
concentrations of triaromatic dinosteroids, dinosterane and 24-norcholestanes, while the middle-upper interval showed the reverse characteristics. Tang and Wang (2007) reported that the Cambrian-Lower Ordovician oils were enriched with gammacerane and $\mathrm{C}_{28}$ sterane and low in diasteranes. All these previous studies are confirmed by our investigation. Among the oils discovered so far in the Tarim Basin, oils from the TD2 $\left(\in-\mathrm{O}_{1}\right)$ and TZ62 (S) wells are suggested to be typical of the Cambrian-Lower Ordovician genetic affinity (Xiao

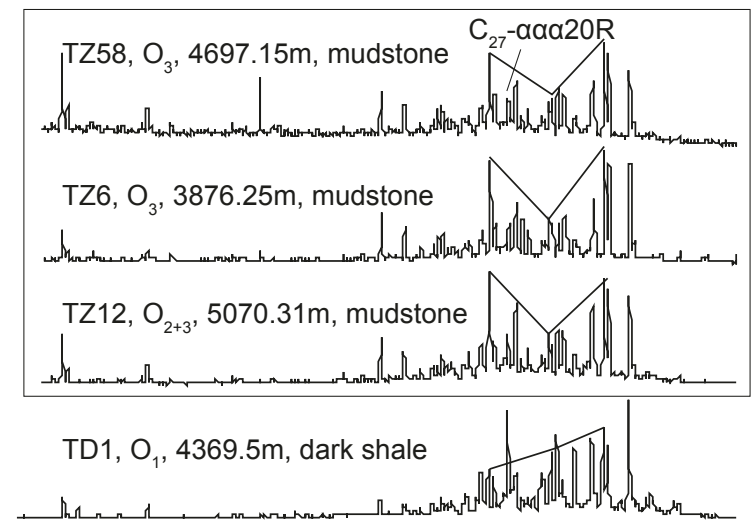

et al, 2005; Tang and Wang, 2007) (Figs. 3 and 5). The oil from YM2 $\left(\mathrm{O}_{1}\right)$ shows good correlation with the $\mathrm{O}_{2+3}$ source rocks according to biomarkers (Figs. 3 and 5), which was also predicted to be derived from the $\mathrm{O}_{2+3}$ source rocks by geological reasoning by a SINOPEC in-house study. We suggest that the TD2 $\left(\in-\mathrm{O}_{1}\right)$ (plus TZ62 $\left.(\mathrm{S})\right)$ and YM2 $\left(\mathrm{O}_{1}\right)$ oils could serve as two end member oils sourced from $\in-\mathrm{O}_{1}$ and $\mathrm{O}_{2+3}$, respectively, based on biomarkers (Figs. 3 and 5).

Detailed oil-oil and oil-source rock correlations show that

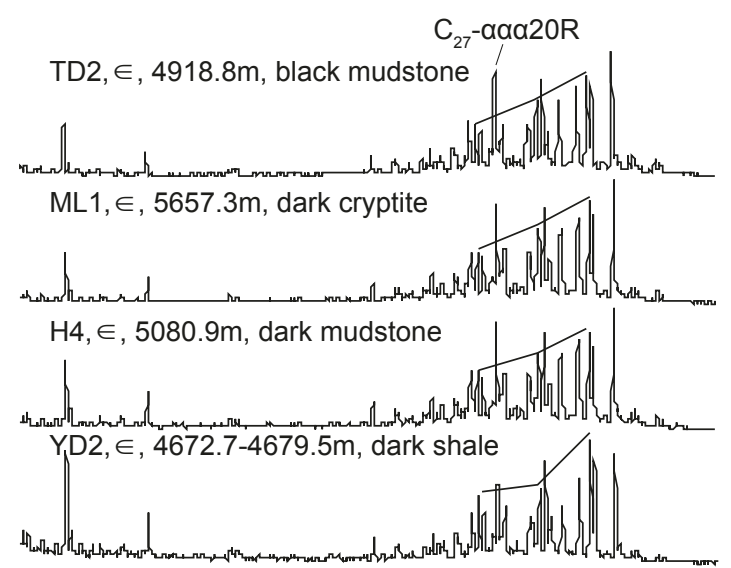

Fig. $5 \mathrm{M} / \mathrm{z} 217$ mass fragmentograms of saturated hydrocarbon fractions of potential source rocks in the Tarim Basin

most Ordovician oils in the Tazhong Uplift display "V-shaped" relative distribution of $\mathrm{C}_{27}, \mathrm{C}_{28}$ and $\mathrm{C}_{29}$ sterane, compared with the oils from the YM2 well and $\mathrm{O}_{2+3}$ mudstones (Figs. 3 and 5). In the Ternary plot showing the relative distribution of $\mathrm{C}_{27}-\mathrm{C}_{28}-\mathrm{C}_{29}$ steranes, most Ordovician oils are plotted with the $\mathrm{O}_{2+3}$ source rocks (Fig. 6). It seems that only a small number of the oils marked with shadow in Fig. 7 have typical characteristics of Cambrian-Lower Ordovician source rocks (e.g. YD2 well) and the associated oils (e.g. TD2 well, Fig. 7, Table 2), including relatively high ratios of gammacerane $/ \mathrm{C}_{30}$ hopane (Fig. 7(b), Table 2), and low ratios of $\mathrm{C}_{29}$ diasterane/ $\mathrm{C}_{29}$ regular sterane (Fig. 7(c), Table 2), $\mathrm{C}_{27} / \mathrm{C}_{29}$ steranes (Table 2) and $\mathrm{C}_{27} / \mathrm{C}_{29} \alpha \beta \beta 20(\mathrm{R}, \mathrm{S})$ (Fig. 7(a)) as well as low sterane isomerization (Fig. 7(d), Table 2) but relatively high abundance of dinosteranes (Fig. 4) and 24-norcholestanes. It seems that most of the Tazhong Ordovician oils are derived from $\mathrm{O}_{2+3}$ source rocks (Figs. 3-7). However, compound specific $n$-alkane stable carbon analysis does not support the biomarker results as discussed in the following section.

\subsubsection{Correlation of compound specific isotopes}

The isotopic composition of $n$-alkane was suggested to be controlled primarily by the source input and depositional paleoenvironment of source rocks (Zhang et al, 1992) with little or only a minor influence from maturity and migration fractionation (Clayton and Bjorøy, 1994). Based on stable carbon isotopic compositions $\left(\delta^{13} \mathrm{C}\right)$ of $n$-alkanes, the Cambrian source rock extracts from the YD2 and the Cambrian sourced oil from the TD2 well (Tang and Wang, 2007) are the most ${ }^{13} \mathrm{C}$ enriched compared with other oils, especially the YM2 oil, which is the most ${ }^{13} \mathrm{C}$ depleted (Fig. 8(b)). The oil from the TZ62 (S) well is also enriched in ${ }^{13} \mathrm{C}$.

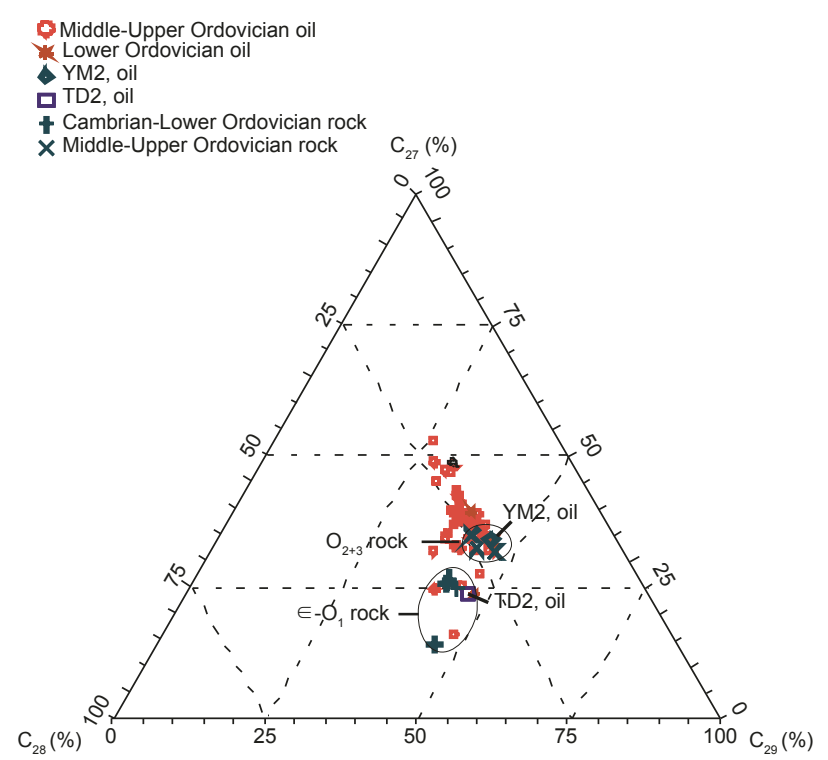

Fig. 6 Ternary plot showing the relative distribution of $\mathrm{C}_{27}-\mathrm{C}_{28}-\mathrm{C}_{29}$ steranes in the Ordovician oils and source rock extracts from the Tarim Basin

The TZ62 (S) pool has previously been recognized as a subtle lenticular oil pool (Wang and Xiao, 2004). The YM2 well is located in the Tabei Uplift in the northern Tarim Basin. Both the biomarkers and $\delta^{13} \mathrm{C}$ values suggest that the TZ62 (S) (and /or TD2 $\left.\left(\in-\mathrm{O}_{1}\right)\right)$ and YM2 $\left(\mathrm{O}_{1}\right)$ oils can serve as two end member oils representing $\in-\mathrm{O}_{1}$ (end member $\mathrm{A}$ ) and $\mathrm{O}_{2+3}$ (end member B).

The $\delta^{13} \mathrm{C}$ values of Tazhong Ordovician oils vary in a wide range (Fig. 8(a)). However, all of these oils do not 
$\mathrm{C}_{27} / \mathrm{C}_{29}$ sterane $\alpha \beta \beta 20(\mathrm{R}, \mathrm{S})$ Gammacerane/C $\mathrm{C}_{30}$-hopane $\mathrm{C}_{29}$-diasterane/C $\mathrm{C}_{29}$-regular sterane $\mathrm{C}_{29}$ sterane $\alpha \beta \beta /(\alpha \alpha \alpha+\alpha \beta \beta)$

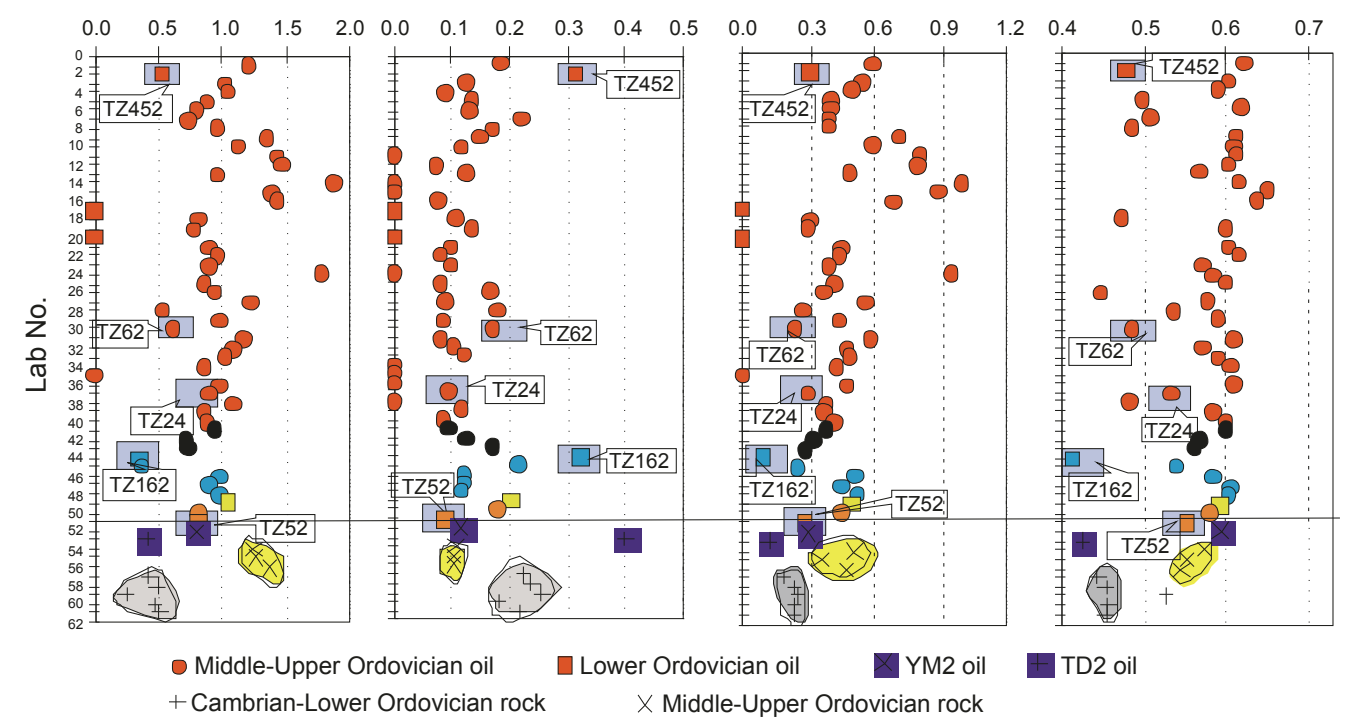

Fig. 7 Oil-oil and oil-source rock correlation showing mixed sources of the Tazhong Ordovician oils

extend beyond the critical envelope of the two end member oils (Fig. 8(b)). The compound specific isotopes of $n$-alkanes show that the majority of the Ordovician oils in the Tazhong Uplift are actually mixed oils from both the Cambrian-Lower Ordovician and the Middle-Upper Ordovician strata, rather than from the $\mathrm{O}_{2+3}$ strata as previous studies have indicated
(Zhang et al, 2000a; 2000b). It is interesting to note that the oils in the shaded portions of Fig. 7, which display characteristics much more like Cambrian-Lower Ordovician genetic affinity oil (based on biomarkers), are also mixed oils according to the $\delta^{13} \mathrm{C}$ of $n$-alkanes measured in this study (Fig. 8).
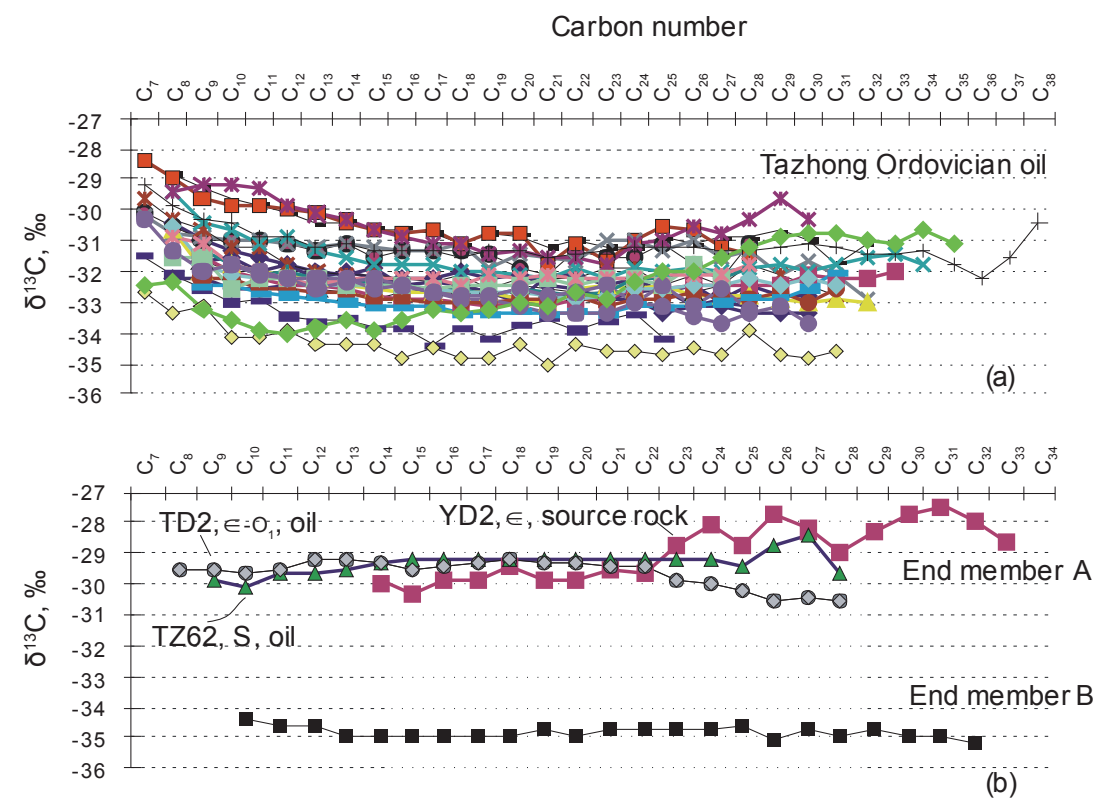

Fig. 8 Compound specific $n$-alkane stable carbon isotope of Ordovician oils in the Tazhong Uplift

\subsection{Quantitative prediction of the mixed oils}

Compound specific isotope analysis of $n$-alkane has been used in quantitative evaluation of mixed gas (Berner and Faber, 1988; Jenden et al, 1993; Jin et al, 2004; Cheng and Jin, 2005). This can be used similarly in the evaluation of mixed oils. The stable carbon isotope ratio of $n \mathrm{C}_{20}$ was selected for predicting the proportion of the oils mixed, which has a stable isotopic value with almost an intermediate carbon number within the range of 8-31. (The carbon number of $n$-alkane is in the range of $\mathrm{C}_{7}-\mathrm{C}_{38}$, and $\mathrm{C}_{20}$ is nearly in the middle of the range. The $\delta^{13} \mathrm{C}$ of $n \mathrm{C}_{20}$ is relatively stable relative to the low or high carbon number in the two ends which may be influenced by the stability of isotopic 
instrument or low concentration of $n$-alkanes.) A formula used in predicting the proportion of the Ordovician mixed source oils is as follows:

$$
m(\%)=\frac{c_{i} \times \delta \mathrm{C}_{i}-c_{\mathrm{Eb}} \times \delta \mathrm{C}_{\mathrm{Eb}}}{c_{\mathrm{Ea}} \times \delta \mathrm{C}_{\mathrm{Ea}}-c_{\mathrm{Eb}} \times \delta \mathrm{C}_{\mathrm{Eb}}} \times 100
$$

in which, $\delta \mathrm{C}_{i}$ is compound specific isotope ratio $\left(n \mathrm{C}_{20}\right)$ of oil $i ; \delta \mathrm{C}_{\mathrm{Ea}}$ is compound specific isotope ratio of end member $\mathrm{A} ; \delta \mathrm{C}_{\mathrm{Eb}}$ is compound specific isotope ratio of end member $\mathrm{B} ; c_{i}, c_{\mathrm{Ea}}, c_{\mathrm{Eb}}$ are concentrations of $n \mathrm{C}_{20}$ in oil $i$, end member oil $\mathrm{A}$ and $\mathrm{B}$, respectively. A rough proportion of the oils mixed can be calculated. The results were illustrated in Fig. 9. It shows that the Cambrian-Lower Ordovician genetic affinity oils mixed in the Tazhong Ordovician oils studied are approximately in the range of $10.8 \%-74.1 \%$, and it is observed that there is an increasing trend of the percentage of $\in-\mathrm{O}_{1}$ derived oils mixed with increasing burial depth (Fig. 9), which suggests that many native reservoirs charged with $\in-\mathrm{O}_{1}$ genetic affinity oils may originally be situated in deep positions with some of them possibly surviving up to now.

\section{Percentage of $\in-\mathrm{O}_{1}$ genetic affinity oil, \%}

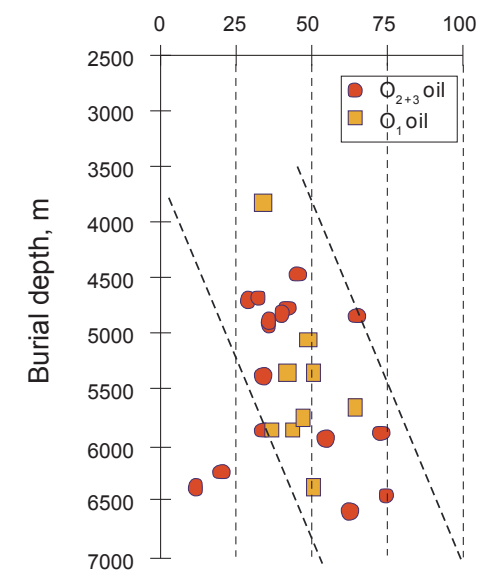

Fig. 9 Percentage of $\in-\mathrm{O}_{1}$ genetic affinity oils mixed in partial Tazhong Ordovician oils versus burial depth

\subsection{Discussion}

There are several types of Ordovician oils in the Tazhong Uplift in terms of physical properties and chemical compositions. These seem to be controlled by the position of a specific tectonic belt. It was observed that condensate oil was primarily located in the No.1 Fault Belt (Li et al, 2008a), whereas the normal or heavy black oils were situated in the TZ47-15 well block (Li et al, 2008b). We believe that the No.1 fault and its associated faults have played an essential role in connecting deep oil sources and the shallow traps. Moreover, multiple oil charging episodes also influence the various oils in the Tazhong Uplift.

The absolute abundance of the biomarkers in oil or rock extracts is obviously controlled by maturity ( $\mathrm{Li}$ et al, 2003; 2005; Pang et al, 2003) and secondary alteration such as migration and biodegradation. It is suggested that there are at least three oil charging episodes in the Tazhong Uplift (Jia,
1995; 1999). The biomarker approach can not be used solely and effectively in discriminating genetic type especially for source rocks for the oils in the area (Liang et al, 2000). For example, if the oil with few or a small number of biomarkers due to high maturity is mixed with normal oil, the mixed oil will only display the biomarker fingerprints of the normal oil. This is the main reason why the results of biomarkers are different from those of the compound specific isotopes in oiloil or oil-source correlation in the Tarim Basin. Therefore, the proportion of the Middle-Upper genetic affinity oils mixed predicted by Zhang et al (2007) based on ratios of $\mathrm{C}_{28} /$ $\left(\mathrm{C}_{27}+\mathrm{C}_{28}+\mathrm{C}_{29}\right)$ regular sterane and gammacerane/ $\mathrm{C}_{31}$ hopane is different from our result by stable carbon isotope in this study.

The potential source rocks of the Middle-Upper Ordovician were proposed to be anoxic marine marl deposited in slope dysoxic or anoxic environments adjacent to a widespread carbonate platform margin (Hanson et al, 2000; Zhang et al, 2000a; 2000b) while the Cambrian-low Ordovician source rocks were deposited under euxinic conditions (Liang et al, 2000; Sun et al, 2003). The differences of the $\delta^{13} \mathrm{C}$ values of the oils (Xiao et al, 2004) derived from Cambrian and Ordovician source rocks may be a result of varying depositional environments. The oils in the Tazhong Uplift might have undergone complicated geological and geochemical actions such as migration fractionation, biodegradation, evaporative fractionation and thermal evolution. Among them maturity may be the most important factor controlling the $\delta^{13} \mathrm{C}$ values of $n$-alkanes in the oils. However, we have recently observed that thermal maturity has little effect on the isotopic composition of $n$ alkanes in rock extracts with a maturity level ranging from low to over high in the Dongying Depression, Bohai Bay Basin. It was also suggested by Clayton and Bjorøy (1994) that the maximum level of a carbon isotope ratio increase caused by maturity is between $2 \%$ - $3 \%$. It is observed that the oil in the Tazhong Uplift which has a higher thermal maturity does not necessarily have a higher $\delta^{13} \mathrm{C}$ value, which suggests that the source is much more important. For instance, the oils from TD2 $\left(\mathrm{O}_{1}\right)$ and TZ62 $(\mathrm{S})$ have the highest $\delta^{13} \mathrm{C}$ values but a middle level of maturity compared with other oils. It seems that there have been no reports that other secondary alterations such as migration and evaporative fractionation can exhibit a strong influence on isotopic composition of $n$-alkanes relative to source and maturity. Further work on this topic is under way.

\section{Conclusions}

Based on biomarkers, most of the Ordovician oils in the Tazhong Uplift show a close genetic relationship with the Middle-Upper Ordovician source rocks. However, the $\delta^{13} \mathrm{C}$ values of the $n$-alkanes in the oils show that most of the oils have intermediate $\delta^{13} \mathrm{C}$ values between the $\in-\mathrm{O}_{1}$ and $\mathrm{O}_{2+3}$ genetic affinity oils, indicating mixed sources. This opinion is different from previous studies suggesting that the $\mathrm{O}_{2+3}$ is the main source rock for the oils. The inconsistency between biomarkers and compound specific isotopes of $n$-alkanes in determining the sources for the Tazhong Ordovician 
oils, reflects multiple hydrocarbon charging episodes and differences of the chemical compositions in each of the episodes. It is observed that there is an increasing trend of the proportion of the $\in-\mathrm{O}_{1}$ genetic affinity oils with increasing burial depth, suggesting that the shorter the distance between the traps and the $\in-\mathrm{O}_{1}$ source rock kitchen, the larger amounts the $\in-\mathrm{O}_{1}$ genetic affinity oils entrapped. The results are significant for deep hydrocarbon exploration in the Tazhong area, and crucial for identifying key source rocks as well as evaluation of hydrocarbon reserves in the Tarim Basin and the similar basins.

\section{Acknowledgements}

This study is funded by the Natural Science Research Council of China (973 State Key Research and Development Program 2006CB202308) and National Natural Science Foundation of China (Grant No.40973031). The authors would like to thank Li Mei, Zhang Haizhu, and Lu Yuhong for sample collection and useful discussion, and Zhao Hong, Chen Zulin, Mao Zhichao of the Yangtze University for excellent technical assistances.

\section{References}

Berner U and Faber E. Maturity related mixing model for methane, ethane and propane, based on carbon isotopes. Organic Geochemistry. 1988. 13(1-3): 67-72

Cai C F, Hu W S and Worden R H. Thermochemical sulphate reduction in Cambro-Ordovician carbonates in Central Tarim. Marine and Petroleum Geology. 2001. 18(6): 729-741

Cai C F, Li H T, Wu G H, et al. Sulfur isotopes as markers of oilsource correlation and thermochemical sulphate reduction in central Tarim. Abstracts. International Meeting on Organic Geochemistry. September 19-24, 2007. Torquay, UK. 297-298

Cai C F, Xie Z Y, Worden R H, et al. Methane-dominated thermochemical sulphate reduction in the Triassic Feixianguan Formation East Sichuan Basin, China: towards prediction of fatal $\mathrm{H}_{2} \mathrm{~S}$ concentrations. Marine and Petroleum Geology. 2004. 21(10): 1265-1279

Chen J H, Fu J M, Sheng G Y, et al. Diamondoid hydrocarbon ratios: novel maturity indices for highly mature crude oils. Organic Geochemistry. 1996. 25(3-4): 179-190

Chen L X, Yang H J, Wu G H, et al. Characteristics of the Ordovician reef-shoal reservoir in Tazhong No.1 slope-break zone, Tarim Basin. Xinjiang Petroleum Geology. 2008. 29(3): 327-330 (in Chinese)

Cheng F Q and Jin Q. A novel approach for quantitative study on twosource-mixed natural gas reservoirs. Acta Sedimentologica Sinica. 2005. 23(3): 554-558 (in Chinese)

Clayton $\mathrm{C} \mathrm{J}$ and Bjorøy M. Effect of maturity on ${ }^{13} \mathrm{C} /{ }^{12} \mathrm{C}$ ratios of individual compounds in North Sea oils. Organic Geochemistry. 1994. 21(6/7): 737-750

Gong S, George S C, Volk H, et al. Petroleum charge history in the Lunnan Low Uplift, Tarim Basin, China-Evidence from oilbearing fluid inclusions. Organic Geochemistry. 2007. 38(8): 13411355

Graham S A, Brassell S, Carroll A R, et al. Characteristics of selected petroleum source rocks, Xinjiang Uygur Autonomous Region, northwest China. AAPG Bulletin. 1990. 74(4): 493-512

Guo J J, Chen J F, Shi S B, et al. Origin and geochemical characteristics of Ordovician oils from Tazhong Uplift. Acta Sedimentologica Sinica. 2009. 27(4): 732-739 (in Chinese)

Han J F, Mei L F, Yang H J, et al. The study of hydrocarbon origin, transport and accumulation in Tazhong area, Tarim Basin. Natural Gas Geoscience. 2007. 18(3): 426-435 (in Chinese)

Han J F, Mei L F, Yang H J, et al. Hydrocarbon accumulation mechanism of large-sized reef-shoal complex condensate gas field in Tazhong No.1 slope-break zone, Tarim Basin. Xinjiang Petroleum Geology. 2008. 29(3): 323-326 (in Chinese)

Hanson A D, Zhang S C, Moldowan J M, et al. Molecular organic geochemistry of the Tarim Basin, northwest China. AAPG Bulletin. 2000. 84(8): 1109-1128

Jenden P D, Drazan D J and Kaplan I R. Mixing of thermogenic natural gases in northern Appalachian Basin. AAPG Bulletin. 1993. 77(6): 980-998

Jia C C. Structural characteristics and oil/gas accumulative regularity in Tarim Basin. Xinjiang Petroleum Geology. 1999. 20(3): 177-183 (in Chinese)

Jia C C. Tectonic Evolution and Regional Structural Geology of Tarim Basin. Beijing: Petroleum Industry Press. 1995 (in Chinese)

Jiang C Q, Li M W, Osadetz K G, et al. Bakken/Madison petroleum systems in the Canadian Williston Basin: Part 2: molecular markers diagnostic of Bakken and Lodgepole source rocks. Organic Geochemistry. 2001. 32(9): 1037-1054

Jin Q, Cheng F Q and Liu W H. Study on source-mixed gas reservoirs and source-mixed ratios. Natural Gas Industry. 2004. 24(2): 22-24 (in Chinese)

Li S M, Pang X Q, Li M W, et al. Geochemistry of petroleum systems in the Niuzhuang south slope of Bohai Bay Basin: Part 1: source rock characterization. Organic Geochemistry. 2003. 34(3): 389-412

Li S M, Pang X Q, Li M W, et al. Geochemistry of petroleum systems in the Niuzhuang south slope of Bohai Bay Basin: Part 4: evidence for new exploration horizons in a maturely explored petroleum province. Organic Geochemistry. 2005. 36(8): 1135-1150

Li S M, Pang X Q, Yang H J, et al. Characteristics and genetic type of the oils in the Tazhong Uplift. Earth Science. 2008a. 33(5): 635-642 (in Chinese)

Li S M, Pang X Q, Yang H J, et al. Geochemical characteristics and implication of high thermal maturity oils in Tazhong-I faulted slope break zone. Oil \& Gas Geology. 2008b. 29(2): 210-216 (in Chinese)

Liang D G, Zhang S C, Zhang B M, et al. Understanding on marine oil generation in China based on Tarim Basin. Earth Science Frontiers. 2000. 7(4): 533-547 (in Chinese)

Lü X X, Jin Z J, Liu L F, et al. Oil and gas accumulations in the Ordovician carbonates in the Tazhong Uplift of Tarim Basin, west China. Journal of Petroleum Science and Engineering. 2004. 41(1/3): 109-121

Luo X Y, Zhao Z J and Meng Y L. The application of n-alkanes odd-even carbon number predominance in oil-source rock correlation-an example of the lower Paleozoic in Tarim Basin. Petroleum Geology \& Experiment. 2007. 29(1): 74-77 (in Chinese)

Pang X Q, Li M W, Li S M, et al. Geochemistry of petroleum systems in the Niuzhuang south slope of Bohai Bay Basin: Part 2: evidence for significant contribution of mature source rocks to "immature oils" in the Bamianhe field. Organic Geochemistry. 2003. 34(7): 931-950

Sun Y G, Xu S P, Lu H, et al. Source facies of the Paleozoic petroleum systems in the Tabei uplift, Tarim Basin, NW China: implications from aryl isoprenoids in crude oils. Organic Geochemistry. 2003. 34(4): 629-634

Tang Y J and Wang T G. Molecular fossils and oil-source rock correlations of Cambrian heavy oil in Tadong 2 well in Tarim Basin. Journal of China University of Petroleum. 2007. 31(6): 18-22 (in Chinese)

Wang Z M and Xiao Z Y. A review of the source rocks for the marine oils in the Tarim Basin. Chinese Science Bulletin. 2004. 49(Supplement): 1-8 (in Chinese)

Wei Z B, Moldowan J M, Zhang S C, et al. Diamondoid hydrocarbons 
as a molecular proxy for thermal maturity and oil cracking: Geochemical models from hydrous pyrolysis. Organic Geochemistry. 2007. 38(2): 227-249

Xiao Z Y, Huang G H, Lu Y H, et al. Origin of oils from well Tadong 2 in Tarim Basin. Acta Sedimentologica Sinica. 2004. 22(Supplement): 66-72 (in Chinese)

Xiao Z Y, Lu Y H, Sang H, et al. A typical Cambrian oil reservoir: Origin of oil reservoir in well TZ62, Tarim Basin. Geochimica. 2005. 34(2): 155-160 (in Chinese)

Zeng J H, Wu Q, Yang H J, et al. Chemical characteristics of formation water in Tazhong area of the Tarim Basin and their petroleum geology significance. Oil \& Gas Geology. 2008. 29(2): 223-229 (in Chinese)

Zhang M, Huang G H, Zhao H J, et al. Quantitative identification pattern and its significance to marine mixed oils in Tarim Basin. Journal of Oil and Gas Technology. 2007. 29(4): 34-50 (in Chinese)

Zhang S C, Hanson A D, Moldowan J M, et al. Paleozoic oil-source rock correlations in the Tarim Basin, NW China. Organic Geochemistry. 2000a. 31(4): 273-286

Zhang S C, Huang H P, Xiao Z Y, et al. Geochemistry of Palaeozoic marine petroleum from the Tarim Basin, NW China: Part 2: Maturity assessment. Organic Geochemistry. 2005. 36(8): 1215-1225
Zhang S C, Liang D G and Zhang B M. Formation of Marine Petroleum in Tarim Basin. Beijing: Petroleum Industry Press. 2004 (in Chinese)

Zhang S C, Zhang B M, Wang F Y, et al. Middle-upper Ordovician: the main source of the oils in the Tarim Basin. Marine Origin Petroleum Geology. 2000b. 5(2): 16-22 (in Chinese)

Zhang T W, Amrani A, Ellis G S, et al. Experimental investigation on thermochemical sulfate reduction by $\mathrm{H}_{2} \mathrm{~S}$ initiation. Geochimica et Cosmochimica Acta. 2008. 72(14): 3518-3530

Zhang W Z, Pei G and Guan D S. Investigation of compound specific isotope of gasoline hydrocarbons from Paleozoic and Mesozoic oils in the Ordos Basin. Chinese Science Bulletin. 1992. 3: 248-251 (in Chinese)

Zhao M J, Qin S F, Pan W Q, et al. The hydrocarbon source analysis of buried-hill reservoir of Ordovician in western Lunnan area of Tabei Uplift. Xinjinag Petroleum Geology. 2008. 29(4): 478-481 (in Chinese)

Zhou X Y, Wang Z M, Yang H J, et al. Cases of discovery and exploration of marine fields in China (Part 5): Tazhong Ordovician condensate field in Tarim Basin. Marine Origin Petroleum Geology. 2006. 11(1): 45-51 (in Chinese) 